\title{
Distribuição das espécies do gênero Anopheles (Diptera, Culicidae) no Estado do Maranhão, Brasil
}

\author{
Distribution of species from genus Anopheles \\ (Diptera, Culicidae) in the State of Maranhão, \\ Brazil
}

\author{
${ }_{1}$ Departamento de Patologia, \\ Universidade Federal do \\ Maranhão, São Luís, Brasil. \\ 2 Fundação Nacional de \\ Saúde, São Luís, Brasil. \\ Correspondência \\ J. M. M. Rebêlo \\ Laboratório de Entomologia \\ e Vetores, Departamento \\ de Patologia, Universidade \\ Federal do Maranhão. \\ Praça Madre Deus 2, \\ São Luís, MA \\ 65025-560, Brasil. \\ macariorebelo@uol.com.br
}

\section{Abstract}

We studied the distribution and diversity of Anopheles species in 123 counties (municipalities) in the State of Maranhão, Brazil. The basic method consisted of capturing female specimens inside and around human dwellings between 6 PM and 6 AM from January 1992 to December 2001. A total of 84,467 specimens belonging to 24 species were captured, with a predominance of A. triannulatus sensu lato $(20,788)$, A. darlingi (19,083), A. nuneztovari $(16,884)$, A. albitarsis s.l. (14,352), A. aquasalis (8.202), and A. evansae $(2,885)$. The other 18 species together accounted for only $2.7 \%$ of the total. The species found in the most counties were A. albitarsis s.l. (109 counties), A. triannulatus s.l. (106), A. nuneztovari (93), A. darlingi (87), and A. evansae (64). The richness and wide distribution of anopheline species in Maranhão agree with the State's geographic position among Brazil's macro-regions, resulting in a mixed fauna with representative elements from these regions.

Anopheles; Malaria; Demography

\author{
José M. Macário Rebêlo ${ }^{1}$ \\ Jorge L. P. Moraes 2 \\ Gildário A. Alves 2 \\ Francisco S. Leonardo 2 \\ Roseno V. da Rocha ${ }^{2}$ \\ Walter Araújo Mendes 2 \\ Elizaldo Costa 2 \\ Lucy E. M. B. Câmara 2 \\ Márcio J. A. Silva 1 \\ Yrla N. O. Pereira 1 \\ José A. C. Mendonça ${ }^{1}$
}

\section{Introdução}

A distribuição das espécies de anofelinos no Estado do Maranhão, Brasil, é pouco estudada. Os primeiros registros foram feitos por Deane et al. 1 e, posteriormente, por Ferreira Neto et al. 2 e Xavier \& Mattos 3. Estudos de levantamentos faunísticos, padronizados, foram realizados mais recentemente nos Municípios de Buriticupu 4 , Raposa 5 e Pinheiro ${ }^{6}$. Embora existam poucos trabalhos publicados, os dados disponíveis até o presente - derivados, sobretudo, de estudos feitos no ambiente modificado, dentro e nos arredores das habitações - revelam a presença de uma fauna diversificada.

Ainda assim, esses dados não representam uma abordagem concludente da fauna anofélica, visto que vários ecossistemas que caracterizam o Maranhão, em face de sua dimensão e posição geográfica de transição, sequer foram estudados. Somente trabalhos sistematizados e duradouros abrangendo todas as fitorregiões poderão fornecer uma amostragem mais fiel da fauna.

Neste trabalho, apresentam-se novos dados sobre os anofelinos, com o intuito de ampliar os conhecimentos sobre sua diversidade e distribuição geográfica no Estado do Maranhão.

\section{Caracterização da área de estudo}

Os anofelinos foram estudados em 123 municípios malarígenos do Estado do Maranhão, distri- 
buídos nas seguintes regiões: noroeste (29 municípios), nordeste (38), sudoeste (28) e central (28) (Figura 1). A seguir, faz-se uma breve descrição sobre as bacias hidrográficas, vegetação e clima de cada região de acordo com o Instituto Brasileiro de Geografia e Estatística (IBGE) 7 .

Os municípios da região noroeste ocupam a área que se estende desde o baixo curso do rio Gurupi, toda a bacia do rio Pericumã Aurá até o baixo curso do rio Pindaré. A cobertura vegetal predominante é a floresta amazônica, todavia, nas partes mais baixas, predominam os campos inundáveis. O clima é o tropical quente e úmido, com índices pluviométricos variando entre $1.800 \mathrm{~mm}$ e $2.200 \mathrm{~mm}$.

Os municípios da região nordeste ocupam toda a bacia do rio Munim e parte das bacias dos rios Parnaíba e Itapecuru. A vegetação é complexa, pois forma um misto de cerrado, caatinga, mata perenifólia aberta, cocal e restinga. Os municípios banhados pelos rios Itapecuru e Munim são influenciados pelo clima semi-úmido de transição entre os climas quente e úmido e o semi-árido, com índices pluviométricos em torno de $1.800 \mathrm{~mm}$. Aqueles situados às margens do rio Parnaíba estão compreendidos na zona semi-árida, com índices pluviométricos anuais oscilando entre $1.000 \mathrm{~mm}$ e $1.600 \mathrm{~mm}$.
A região central é mais conhecida como a zona dos cocais, devido à presença freqüente da palmeira babaçu (Attalea speciosa Mart. Ex. Spreng.) associada com a mata perenifólia aberta. Os municípios que ocupam a bacia do rio Mearim, no seu médio curso, são influenciados pelo clima tropical quente-úmido; aqueles situados na bacia do rio Itapecuru recebem influência do clima semi-úmido. Os índices pluviométricos variam de $1.200 \mathrm{~mm}$ a $1.600 \mathrm{~mm}$.

Na região sudoeste, a área que se estende do alto curso do rio Gurupi ao médio curso do rio Pindaré recebe influência do clima tropical quente-úmido e apresenta índices pluviométricos de $1.600 \mathrm{~mm}$, tendo como cobertura vegetal predominante a floresta amazônica. Na área do baixo curso do rio Tocantins e alto curso do rio Grajaú e na confluência das bacias do Tocantins, Mearim e Parnaíba, predominam o cerrado e o clima semi-úmido, com índices pluviométricos variando de $1.000 \mathrm{~mm}$ a $1.400 \mathrm{~mm}$.

\section{Metodologia}

Os anofelinos foram capturados em horários variáveis entre $18 \mathrm{~h}$ e $6 \mathrm{~h}$, mensalmente, durante dez anos, de janeiro de 1992 a dezembro de 2001. O
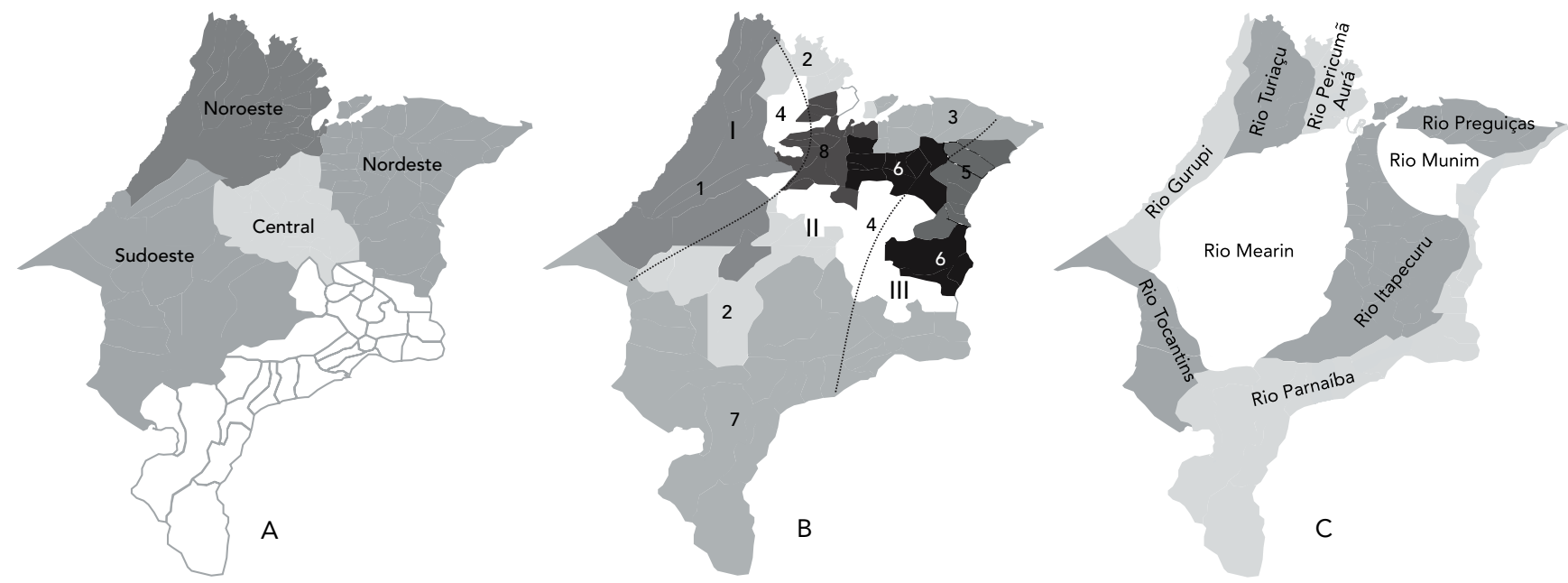

1: floresta estacional perenifólia densa; 2: floresta estacional perenifólia aberta; 3: dunas, restinga, mangue e cerrado; 4: floresta estacional perenifólia aberta com babaçu; 5: cerrados e caatingas; 6: floresta estacional perenifólia aberta com babaçu e manchas de cerrado; 7: cerrados meridionais; 8: campo aluvial flúvio-marinho. Clima: I. quente-úmido; II. semi-úmido e III. semi-árido. 
total de horas trabalhadas em cada região foi o seguinte: noroeste (4.023), sudoeste (3.802), central (2.248) e nordeste (1.851).

As coletas eram feitas com tubo de sucção, semelhante ao aspirador de Castro, dentro das habitações humanas, no peridomicílio - até 30 metros das casas - e no extradomicílio - margens de rios, riachos, córregos, igarapés, lagoas e açudes distantes mais de 30 metros das habitações. Estas eram, na sua maioria, rurais, construídas de barro batido e cobertas de palha.

Os exemplares coletados eram mortos em câmaras de acetato de etila, acondicionados entre papel absorvente no interior de placas de Petri de polietileno e transportados para o laboratório. A identificação taxonômica foi feita pelos técnicos de entomologia da Fundação Nacional de Saúde nos laboratórios regionais, com posterior revisão no Laboratório Central, em São Luís, com auxílio das chaves dicotômicas propostas por Corrêa \& Ramalho (1958, apud Forattini ${ }^{8}$ ) e por Consoli \& Lourenço-de-Oliveira 9 . Sabe-se que muitas espécies de anofelinos neotropicais fazem parte de complexos de espécies crípticas. Considerando que, neste estudo, não foi feita distinção das espécies, no seu respectivo complexo, quando for o caso, ao nome específico de cada uma delas será acrescentada a expressão sensu lato (s.l.).

\section{Resultados}

No total, foram encontradas 24 espécies de anofelinos no Estado do Maranhão: Anopheles albitarsis s.l., A. antunesi, A. apicimacula, A. aquasalis, A. argyritarsis, A. brasiliensis, A. darlingi, A. eiseni, A. evansae, A. galvaoi, A. intermedius, A. maculipes, A. mediopunctatus, A. minor, A. nuneztovari, A. oswaldoi, A. peryassui, A. pseudomaculipes, A. rangeli, A. rondoni, A. strodei, $A$. shannoni, A. triannulatus s.l. e Anopheles sp., a qual não foi identificada taxonomicamente por constituir-se de espécimes com características muito próximas às de $A$. benarrochi, facilmente confundível com A. aquasalis e A. galvaoi. Tais problemas poderão ser resolvidos, no futuro, com a observação de caracteres também em outros estágios e sexo, como no ovo, nas larvas e pupas, e na genitália masculina.

Essas espécies estavam distribuídas nas seguintes regiões: noroeste (18 espécies), nordeste (20), sudoeste (15) e central (8) (Tabelas 1, 2, 3 e 4). As espécies A. antunesi, A. apicimacula e $A$. shannoni só foram encontradas na região nordeste (Tabela 2), enquanto Anopheles sp. e A. rondoni só ocorreram na região sudoeste (Tabela 3). As demais se distribuíram em mais de uma região; destas, A. eiseni e $A$. rangeli só foram encontradas nas regiões noroeste e nordeste.

As espécies que se destacaram por ocorrer em maior número de municípios foram A. albitarsis s.l. (109 municípios), A. triannulatus s.l. (106), A. nuneztovari (93), A. darlingi (87) e A. evansae (64) (Tabelas 1, 2, 3 e 4).

Os municípios que apresentaram maior diversidade de espécies foram Barreirinhas e Pinheiro (12 espécies); Imperatriz (11); Nina Rodrigues (10); Turilândia, Anapurus, Presidente Vargas e Urbano Santos (9); Icatu e Santa Rita (8). Nos demais municípios, foram encontradas, no máximo, sete espécies (Tabelas 1, 2, 3 e 4).

Do ponto de vista climático e vegetacional, as espécies encontradas ficaram assim distribuídas: 18 ocorreram na zona quente-úmida caracterizada pelo domínio da floresta amazônica; 20 foram registradas na zona semi-úmida, com um misto de mata/cocal/cerrado/campo inundável, e apenas 11 foram registradas na zona semi-árida, caracterizada pela mistura de mata/cerrado/caatinga (Figura 1).

No total, foram capturados 84.467 exemplares. A região que contribuiu com maior número de indivíduos foi a central (30.654), seguida pela noroeste (23.252), sudoeste (20.284) e nordeste (10.277). Considerando que as regiões diferiram em números de horas trabalhadas, para efeito de correção dessa diferença, fez-se o cálculo da média de indivíduos capturados por hora. Mesmo assim, a ordem das freqüências não diferiu entre as regiões: central (13,6 indivíduos/hora), noroeste (5,8 indivíduos/hora), sudoeste (5,3 indivíduos/hora) e a nordeste (5,5 indivíduos/hora).

A espécie mais freqüente foi $A$. triannulatus s.l. (20.788 espécimes), seguida por $A$. darlingi (19.083), A. albitarsis s.l. (14.352), A. nuneztovari (16.884), A. aquasalis (8.202) e A. evansae (2.885). Essas espécies representaram $97,3 \%$ dos espécimes capturados, contra $2,7 \%$ das outras 18 espécies juntas. A. triannulatus s.l. predominou em duas regiões, sendo superada por A. aquasalis no nordeste e apresentou números semelhantes ao de A. darlingi no noroeste (Tabelas 1, 2, 3 e 4).

\section{Discussão}

O número de espécies de anofelinos encontrado no Estado do Maranhão representa aproximadamente $43,6 \%$ da fauna brasileira (55 espécies). Esses dados concordam com a posição geográfica do estado, entre as macrorregiões que caracterizam o Brasil, resultando em uma fauna mista, com elementos representativos dessas regiões. Para se ter uma noção dessa situação, as espécies encontradas neste estudo representam $72,7 \%$ da 
Distribuição das espécies de anofelinos na região noroeste do Estado do Maranhão, Brasil, de 1993 a 2002.

\begin{tabular}{|c|c|c|c|c|c|c|c|c|c|c|c|}
\hline \multirow[t]{2}{*}{ Municípios } & \multicolumn{11}{|c|}{ Espécies do gênero Anopheles } \\
\hline & albitarsis & aquasalis & argyristarsis & darlingi & eiseni & evansae & galvaoi & intermedius & s maculipes & $\begin{array}{l}\text { medio- } \\
\text { punctatus }\end{array}$ & minor \\
\hline Alcântara & - & 9 & - & - & - & - & - & - & - & - & - \\
\hline Amapá do Maranhão & - & - & - & - & - & - & - & - & - & - & - \\
\hline Apicum-Açu & 25 & 1.136 & - & 1 & - & - & - & - & 1 & - & - \\
\hline Araguanã & 48 & - & - & 125 & - & - & - & 85 & - & - & - \\
\hline Boa Vista do Gurupi & 144 & - & - & 88 & - & - & - & - & - & - & 2 \\
\hline Cajari & 31 & - & - & 70 & - & 3 & - & - & - & - & - \\
\hline Cândido Mendes & 38 & 606 & - & - & - & - & - & - & - & - & - \\
\hline Carutapera & 6 & 61 & - & - & - & 13 & - & - & - & - & 18 \\
\hline Cedral & - & 218 & - & - & - & - & - & - & - & - & - \\
\hline Centro Novo & 50 & - & - & 24 & - & 1 & - & 22 & - & - & - \\
\hline Cururupu & 2 & 32 & - & - & - & - & - & - & - & - & - \\
\hline Godofredo Viana & 16 & 132 & - & 18 & - & - & - & - & - & - & 4 \\
\hline Governador Nunes Freire & 80 & - & - & 155 & - & 17 & - & 143 & - & - & - \\
\hline Guimarães & - & 1.003 & - & - & - & - & - & - & 1 & - & - \\
\hline Maracaçumé & 284 & - & - & 239 & - & 7 & - & 13 & - & - & - \\
\hline Monção & 681 & 175 & - & 851 & - & 1 & - & 1 & - & - & - \\
\hline Nova Olinda & 34 & - & - & 21 & - & - & - & - & - & - & - \\
\hline Odorico Mendes & 3 & 43 & - & - & - & - & - & - & - & - & - \\
\hline Penalva & - & - & - & 187 & - & - & - & - & - & - & - \\
\hline Pindaré & 1.093 & - & - & 818 & - & 25 & - & 2 & - & - & - \\
\hline Pinheiro & 53 & 119 & 823 & 744 & - & 1 & 103 & - & 1 & 8 & - \\
\hline Presidente Sarney & 77 & - & - & 282 & 1 & - & - & - & - & - & - \\
\hline Santa Helena & 91 & 185 & - & 179 & - & - & - & - & - & - & - \\
\hline Santa Luzia do Paruá & - & - & - & 32 & - & - & - & - & - & - & - \\
\hline Serrano do Maranhão & 20 & 78 & - & 3 & - & - & - & - & - & - & - \\
\hline Turiaçu & 93 & - & - & 57 & - & - & - & - & - & - & \\
\hline Turilândia & 942 & - & - & 1.028 & - & - & - & - & 1 & - & - \\
\hline Vitória do Mearim & 451 & - & - & 821 & - & - & - & - & - & - & - \\
\hline Zé Doca & 71 & - & - & 63 & - & 77 & - & - & - & - & - \\
\hline Total de indivíduos & 4.333 & 3.797 & 823 & 5.806 & 1 & 145 & 103 & 266 & 4 & 8 & 24 \\
\hline Total de municípios & 23 & 13 & 1 & 21 & 1 & 9 & 1 & 6 & 4 & 1 & 3 \\
\hline
\end{tabular}

(continua)

fauna de anofelinos da Amazônia (33 espécies) $1,10,11$, e cerca de $45 \%$ das espécies presentes no Maranhão ocorrem também no Nordeste 12. Todavia, várias espécies têm ampla distribuição no Brasil, ocorrendo desde a Amazônia até o Rio Grande do Sul 13,14,15.

Os estudos sistematizados, com coletas regulares em pontos fixos, previamente realizados nos municípios de Buriticupu 4, Pinheiro ${ }^{6}$ e Raposa 5 , haviam determinado a existência de 12 espécies de anofelinos, mas nenhum notificou a presença de A. antunesi, A. apicimacula, A. eiseni, A. intermedius, A. maculipes, $A$. mediopunctatus, A. minor, A. peryassui, A. pseudomaculipes e $A$. shannoni, todas encontradas no presente estudo. Este resultado mostra a necessidade de se ampliar, cada vez mais, a área dos inquéritos entomológicos, incorporando novos pontos de coleta, inclusive nas áreas silvestres, onde, provavelmente, encontrar-se-ão outras espécies.

As formas de A. albitarsis s.l. e A. triannulatus s.l. presentes no Maranhão não foram determinadas. Sabe-se, no entanto, que $A$. triannulatus s.l. compreende um complexo do qual participam pelo menos três formas diferenciadas morfológica e isoenzimaticamente 16. Recentemente, foi descrita uma nova espécie desse complexo, A. halophylus 17 . Considerando que a A. triannulatus típica é a mais amplamente distribuído, a chance de ela ocorrer no Maranhão seria maior do que a das outras, as quais estão mais associadas à região do Brasil Central e não se relacionam 
Distribuição das espécies de anofelinos na região noroeste do Estado do Maranhão, Brasil, de 1993 a 2002.

\begin{tabular}{|c|c|c|c|c|c|c|c|c|c|}
\hline \multirow[t]{2}{*}{ Municípios } & \multicolumn{7}{|c|}{ Espécies do gênero Anopheles } & \multicolumn{2}{|c|}{ Total } \\
\hline & nuneztovari & oswaldoi & $\begin{array}{l}\text { peryassui- } \\
\text { maculipes }\end{array}$ & pseudo & rangeli & strodei & triannulatus & Indivíduos & Espécies \\
\hline Alcântara & - & - & - & - & - & - & - & 9 & 1 \\
\hline Amapá do Maranhão & 3 & - & - & - & - & - & 3 & 6 & 2 \\
\hline Apicum-Açu & - & - & 1 & - & - & - & 68 & 1.232 & 6 \\
\hline Araguanã & 72 & 1 & - & - & - & - & 146 & 477 & 6 \\
\hline Boa Vista do Gurupi & 24 & 1 & - & - & - & - & 56 & 315 & 6 \\
\hline Cajari & 14 & - & - & - & - & - & 103 & 221 & 5 \\
\hline Cândido Mendes & - & - & - & - & - & - & 5 & 649 & 3 \\
\hline Carutapera & 2 & - & - & - & - & - & - & 100 & 5 \\
\hline Cedral & - & - & - & - & - & - & - & 218 & 1 \\
\hline Centro Novo & 28 & 66 & - & - & - & - & 71 & 262 & 7 \\
\hline Cururupu & - & 1 & 1 & 1 & - & - & 13 & 50 & 6 \\
\hline Godofredo Viana & 7 & - & - & - & - & - & 48 & 225 & 6 \\
\hline Governador Nunes Freire & 94 & 3 & - & - & - & - & 139 & 631 & 7 \\
\hline Guimarães & - & - & 9 & - & - & - & - & 1.013 & 3 \\
\hline Maracaçumé & 85 & 21 & - & - & - & - & 96 & 745 & 7 \\
\hline Monção & 574 & - & - & - & - & - & 1.598 & 3.881 & 7 \\
\hline Nova Olinda & 21 & 4 & - & - & - & - & 42 & 122 & 5 \\
\hline Odorico Mendes & - & - & - & - & - & - & - & 46 & 2 \\
\hline Penalva & 6 & - & - & - & - & - & 39 & 232 & 3 \\
\hline Pindaré & 487 & 3 & - & - & - & - & 1.398 & 3.826 & 7 \\
\hline Pinheiro & 16 & 5 & - & - & - & 1 & 9 & 1.883 & 12 \\
\hline Presidente Sarney & 190 & - & - & 4 & - & - & 157 & 711 & 6 \\
\hline Santa Helena & 75 & 1 & - & - & - & 1 & 101 & 633 & 7 \\
\hline Santa Luzia do Paruá & 6 & - & - & - & - & - & 13 & 51 & 3 \\
\hline Serrano do Maranhão & - & 4 & - & - & - & 1 & 13 & 119 & 6 \\
\hline Turiaçu & - & - & - & - & - & - & - & 150 & 2 \\
\hline Turilândia & 208 & 9 & - & 2 & 1 & 5 & 132 & 2.328 & 9 \\
\hline Vitória do Mearim & 403 & - & - & - & - & - & 1.019 & 2.694 & 4 \\
\hline Zé Doca & 88 & 10 & - & - & - & - & 114 & 423 & 6 \\
\hline Total de indivíduos & 2.403 & 129 & 11 & 7 & 1 & 8 & 5.383 & 23.252 & 18 \\
\hline Total de municípios & 20 & 13 & 3 & 3 & 1 & 4 & 23 & 29 & - \\
\hline
\end{tabular}

com a transmissão de malária 16. O A. albitarsis s.l. também é um complexo formado por, pelo menos, três espécies: A. marajoara; A. deaneorum e A. albitarsis sensu stricto 16,18,19,20,21. Parece que, nesse complexo, foi reconhecida uma quarta espécie designada provisoriamente A. albitarsis “B”, que pode ser identificada usando a amplificação polimórfica aleatória do DNA (RAPD) ${ }^{20}$. A. deaneorum é incriminado como vetor no Oeste do Brasil, enquanto A. marajoara é um comprovado vetor primário na Região Nordeste do país.

Quanto a A. nuneztovari, estudos morfológicos, comportamentais, citogenéticos, isoenzimáticos e de DNA mitocondrial 22,23,24 parecem evidenciar a existência de duas espécies crípticas: uma distribuída no Oeste da Venezuela e Co- lômbia e a outra no Brasil. Esta última tem sido descrita como uma espécie muito comum na Região Amazônica, mas sua distribuição estende-se até o Estado do Piauí 10, onde é menos freqüente. Esse padrão de distribuição concorda com as observações levadas a efeito no Estado do Maranhão, pois a densidade dessa espécie é bem mais elevada no lado amazônico do estado (regiões noroeste e sudoeste), do que no lado nordestino, onde a população diminui sensivelmente.

As espécies A. albitarsis s.l., A. triannulatus s.l., A. nuneztovari, A. darlingi, A. evansae, A. oswaldoi e A. galvaoi constituem um grupo de anofelinos com vasta distribuição no território maranhense, ocorrendo juntas em muitos municípios. Alguns autores têm chamado a aten- 
Distribuição das espécies de anofelinos na região nordeste do Estado do Maranhão, Brasil, de 1993 a 2002.

\begin{tabular}{|c|c|c|c|c|c|c|c|c|c|c|c|}
\hline \multirow[t]{2}{*}{ Municípios } & \multirow[b]{2}{*}{ albitarsis } & \multicolumn{10}{|c|}{ Espécies do gênero Anopheles } \\
\hline & & antunesi & $\begin{array}{c}\text { apicima- } \\
\text { cula }\end{array}$ & aquasalis & $\begin{array}{l}\text { argyri- } \\
\text { tarsis }\end{array}$ & brasiliensis & darlingi & eiseni & evansae & galvaoi & $\begin{array}{l}\text { inter- } \\
\text { medius }\end{array}$ \\
\hline Água Doce do Maranhão & 17 & - & - & - & - & - & - & - & 95 & - & - \\
\hline Anajatuba & 566 & - & - & - & - & - & - & - & 12 & - & - \\
\hline Anapurus & 12 & - & - & - & - & 11 & - & - & 36 & - & - \\
\hline Araioses & 5 & - & - & 15 & - & - & - & - & 13 & - & - \\
\hline Bacabeira & 120 & - & - & 239 & - & - & - & - & - & - & - \\
\hline Barreirinhas & 12 & - & 1 & 232 & 1 & 9 & - & - & 1 & - & 2 \\
\hline Belágua & - & - & - & - & - & - & - & - & - & - & - \\
\hline Brejo & 11 & - & - & - & - & - & 16 & - & 8 & - & - \\
\hline Buriti & 14 & - & - & - & - & - & - & - & 13 & - & - \\
\hline Cantanhede & 209 & - & - & - & - & - & 33 & - & 12 & - & - \\
\hline Caxias & 2 & - & - & 2 & - & - & 2 & - & - & - & - \\
\hline Chapadinha & 69 & - & - & - & 2 & - & 41 & - & 11 & - & - \\
\hline Codó & - & - & - & - & - & - & - & - & - & - & - \\
\hline Humberto de Campos & 1 & - & - & 154 & - & - & - & - & 17 & 20 & - \\
\hline Icatu & 7 & - & - & 583 & - & 1 & - & 1 & 5 & 3 & - \\
\hline Itapecuru Mirim & 96 & - & - & - & - & - & 30 & - & 13 & - & - \\
\hline Magalhães de Almeida & 2 & - & - & - & - & - & - & - & - & - & - \\
\hline Mata Roma & 3 & - & - & - & - & 12 & 33 & - & - & - & - \\
\hline Matões & 2 & - & - & - & - & - & 2 & - & 11 & - & - \\
\hline Milagre & 115 & - & - & - & - & - & - & - & 36 & - & - \\
\hline Miranda do Norte & 73 & - & - & - & - & - & - & - & 6 & - & - \\
\hline Nina Rodrigues & 138 & 2 & - & - & - & 1 & 1 & - & 32 & - & 11 \\
\hline Paço do Lumiar & - & 5 & - & 175 & - & - & - & - & - & 4 & - \\
\hline Paulino Neves & 12 & - & - & - & - & - & - & - & - & - & - \\
\hline Pirapemas & 78 & - & - & - & - & - & 25 & - & 59 & - & - \\
\hline Presidente Vargas & 10 & - & - & - & - & - & 1 & - & 25 & - & 76 \\
\hline Primeira Cruz & 15 & - & - & 40 & - & - & - & - & - & - & - \\
\hline Raposa & 95 & - & - & 882 & - & - & - & - & 56 & 139 & - \\
\hline Santana & 2 & - & - & - & - & - & - & - & 136 & - & - \\
\hline Santa Quitéria & 69 & - & - & - & - & - & 20 & - & 33 & - & - \\
\hline Santa Rita & 3 & - & - & - & - & - & - & - & 28 & 1 & 3 \\
\hline São Luís & 2 & - & - & 1.867 & 41 & - & - & - & 31 & 31 & - \\
\hline São Benedito do Rio Preto & - & - & - & - & - & 3 & - & - & - & - & - \\
\hline São Bernardo & 185 & - & - & - & - & - & - & - & 18 & - & - \\
\hline São José de Ribamar & 10 & - & - & 208 & - & - & - & - & 1 & - & - \\
\hline Tutóia & 13 & - & - & 8 & - & - & 3 & - & 2 & - & - \\
\hline Urbano Santos & 6 & - & - & - & - & 1 & - & - & 47 & - & 1 \\
\hline Vargem Grande & 134 & - & - & - & 2 & - & 11 & - & 8 & - & - \\
\hline Números de indivíduos & 2.108 & 7 & 1 & 4.405 & 46 & 38 & 219 & 1 & 765 & 198 & 93 \\
\hline Números de municípios & 34 & 2 & 1 & 12 & 4 & 7 & 14 & 1 & 28 & 6 & 5 \\
\hline
\end{tabular}

(continua)

ção para as similaridades morfológicas entre $A$. galvaoi, A. evansae e A. aquasalis, e, de acordo com Sallum et al. 25, identificar essas espécies pelas fêmeas pode ser problemático. Contudo, $A$ aquasalis constitui uma espécie muito comum no Maranhão e sua área de distribuição no es- tado restringe-se ao litoral, o que explica a ocorrência de inúmeros casos anuais de malária nessa região 5. Já A. galvaoi é encontrada principalmente no interior, sendo poucos os municípios litorâneos onde ocorre junto com A. aquasalis. Segundo Faran 26, A. galvaoi ocorre nos estados 
Distribuição das espécies de anofelinos na região nordeste do Estado do Maranhão, Brasil, de 1993 a 2002.

\begin{tabular}{|c|c|c|c|c|c|c|c|c|c|c|c|}
\hline \multirow[t]{2}{*}{ Municípios } & \multicolumn{9}{|c|}{ Espécies do gênero Anopheles } & \multicolumn{2}{|c|}{ Total } \\
\hline & maculipes & $\begin{array}{c}\text { medio- } \\
\text { punctatus }\end{array}$ & minor & nuneztovari & oswaldoi & peryassui & rangeli & shannoni & $\begin{array}{c}\text { triannu- } \\
\text { latus }\end{array}$ & Indivíduos & Espécies \\
\hline Água Doce do Maranhão & - & - & - & - & - & - & - & - & - & 112 & 2 \\
\hline Anajatuba & - & - & - & 5 & 2 & - & - & - & 168 & 753 & 5 \\
\hline Anapurus & - & 20 & - & 7 & 11 & 20 & 15 & - & 38 & 170 & 9 \\
\hline Araioses & - & - & - & - & - & - & - & - & - & 33 & 3 \\
\hline Bacabeira & - & - & - & - & - & - & - & - & 39 & 398 & 3 \\
\hline Barreirinhas & 1 & - & - & 6 & - & - & 6 & 2 & 5 & 278 & 12 \\
\hline Belágua & - & 14 & - & - & - & 8 & - & - & - & 22 & 2 \\
\hline Brejo & - & - & - & 10 & 3 & - & - & - & 4 & 52 & 6 \\
\hline Buriti & - & - & - & 47 & - & - & - & - & 36 & 110 & 4 \\
\hline Cantanhede & - & - & - & 31 & - & - & - & - & 34 & 319 & 5 \\
\hline Caxias & - & - & - & - & - & - & - & - & 13 & 19 & 4 \\
\hline Chapadinha & - & - & - & 31 & 14 & - & - & - & 204 & 372 & 7 \\
\hline Codó & - & - & - & 8 & - & - & - & - & 30 & 38 & 2 \\
\hline Humberto de Campos & - & 4 & 115 & - & - & - & - & - & 9 & 320 & 7 \\
\hline Icatu & - & - & - & - & - & - & 6 & - & 8 & 614 & 8 \\
\hline Itapecuru Mirim & - & - & - & 15 & 3 & - & - & - & 53 & 210 & 6 \\
\hline Magalhães de Almeida & - & - & - & - & - & - & - & - & - & 2 & 1 \\
\hline Mata Roma & - & 4 & - & 8 & - & 3 & - & - & - & 63 & 6 \\
\hline Matões & - & - & - & 7 & 1 & - & - & - & 5 & 28 & 6 \\
\hline Milagre & - & - & - & 113 & - & - & - & - & 40 & 304 & 4 \\
\hline Miranda do Norte & - & - & - & 18 & - & - & - & - & 5 & 102 & 4 \\
\hline Nina Rodrigues & - & - & - & 134 & 16 & - & 8 & - & 45 & 388 & 10 \\
\hline Paço do Lumiar & - & - & - & - & - & - & - & - & 15 & 199 & 4 \\
\hline Paulino Neves & - & - & - & - & - & - & - & - & & 12 & 1 \\
\hline Pirapemas & - & - & - & 67 & 15 & - & - & - & 117 & 361 & 6 \\
\hline Presidente Vargas & - & 3 & - & 77 & 38 & - & 36 & - & 44 & 310 & 9 \\
\hline Primeira Cruz & - & - & - & - & - & - & - & - & & 55 & 2 \\
\hline Raposa & - & - & - & 5 & - & - & 3 & - & 35 & 1.215 & 7 \\
\hline Santana & - & - & - & - & - & - & - & - & 2 & 140 & 3 \\
\hline Santa Quitéria & - & - & - & 67 & 2 & - & - & - & 8 & 199 & 6 \\
\hline Santa Rita & - & - & - & 7 & 3 & - & 2 & - & 64 & 111 & 8 \\
\hline São Luís & - & 2 & - & 7 & - & - & 2 & - & 149 & 2.132 & 9 \\
\hline São Benedito do Rio Preto & - & - & - & 1 & - & 1 & - & 3 & 4 & 12 & 5 \\
\hline São Bernardo & - & - & - & 30 & - & - & - & - & 20 & 254 & 5 \\
\hline São José de Ribamar & - & - & - & - & - & - & - & - & 10 & 229 & 4 \\
\hline Tutóia & - & - & - & 2 & - & - & - & - & 1 & 29 & 6 \\
\hline Urbano Santos & - & 1 & - & 1 & 1 & 5 & - & - & 2 & 65 & 9 \\
\hline Vargem Grande & - & - & - & 18 & 5 & & - & - & 69 & 247 & 7 \\
\hline Números de indivíduos & 1 & 48 & 115 & 722 & 114 & 37 & 78 & 5 & 1.276 & 10.277 & 20 \\
\hline Números de municípios & 1 & 7 & 1 & 25 & 13 & 5 & 8 & 2 & 31 & 38 & - \\
\hline
\end{tabular}

do Acre, Amazonas, Rondônia, Mato Grosso, Pará e São Paulo, e tem sido registrada também no Paraná 27 e Amapá 28 . Tal padrão de distribuição no Brasil não excluiria a sua presença no Maranhão, sobretudo pela sua ocorrência no vizinho Estado do Pará.
O grupo geográfico formado por A. aquasalis, A. eiseni, A. mediopunctatus e A. rangeli distribuiu-se apenas em municípios das regiões noroeste e nordeste. Esse padrão de distribuição é compreensível com relação à primeira espécie, pois mostra-se condicionado à presença de cria- 
Distribuição das espécies de anofelinos na região sudoeste do Estado do Maranhão, Brasil, de 1993 a 2002.

\begin{tabular}{|c|c|c|c|c|c|c|c|c|}
\hline \multirow[t]{2}{*}{ Municípios } & \multicolumn{8}{|c|}{ Espécies do gênero Anopheles } \\
\hline & albitarsis & argyritarsis & brasiliensis & darlingi & evansae & galvaoi & maculipes & minor \\
\hline Açailândia & 89 & - & - & 98 & 44 & - & - & - \\
\hline Amarante & 317 & - & - & 1.149 & 190 & - & - & - \\
\hline Arame & 32 & - & - & 93 & 155 & - & - & - \\
\hline Barra do Corda & 299 & - & - & 140 & - & - & - & - \\
\hline Bom Jardim & 97 & - & - & 671 & 27 & - & 1 & 12 \\
\hline Bom Jesus das Selvas & 15 & - & - & 125 & 3 & - & - & - \\
\hline Buriticupu & 2 & - & - & 274 & 44 & - & - & - \\
\hline Buritirana & 97 & - & - & 8 & 22 & - & - & - \\
\hline Campestre & 107 & - & - & 203 & - & - & - & - \\
\hline Carolina & - & - & - & 93 & - & - & - & - \\
\hline Cidelândia & 2 & - & - & 2 & 1 & - & - & - \\
\hline Davinópolis & 12 & - & - & 22 & 8 & - & - & - \\
\hline Estreito & 13 & - & - & 46 & 2 & - & - & - \\
\hline Formosa Serra Negra & 12 & - & - & 33 & 3 & - & - & - \\
\hline Governador Edson Lobão & 16 & - & - & 164 & - & - & - & - \\
\hline Grajaú & 116 & - & - & 215 & 94 & - & - & - \\
\hline Imperatriz & 1.374 & 4 & 1 & 1.244 & 761 & 3 & - & - \\
\hline Itinga & 88 & - & - & 219 & 5 & - & - & - \\
\hline João Lisboa & 18 & - & - & 6 & - & - & - & - \\
\hline Montes Altos & 17 & - & - & 17 & 2 & - & - & - \\
\hline Porto Franco & 83 & - & - & - & - & - & - & - \\
\hline Ribamar Fiquene & 113 & - & - & 108 & 4 & - & - & - \\
\hline São Francisco do Brejão & 11 & - & - & 8 & - & - & - & - \\
\hline São João do Paraíso & - & - & - & - & - & - & - & - \\
\hline São Pedro da Água Branca & 115 & - & - & 199 & 142 & - & - & - \\
\hline São Pedro dos Crentes & 47 & - & - & 6 & 72 & - & - & - \\
\hline Senador La Roque & 24 & - & - & 140 & 8 & - & - & - \\
\hline Sítio Novo & 3 & - & & 33 & 15 & - & - & - \\
\hline Total de indivíduos & 3.119 & 4 & 1 & 5.316 & 1.607 & 3 & 1 & 12 \\
\hline Total de municípios & 26 & 1 & 1 & 26 & 21 & I & 1 & 1 \\
\hline
\end{tabular}

(continua)

douros com água salobra, o que explica a sua ocorrência somente nos municípios litorâneos e naqueles cujos corpos d'água têm influência marinha, compreendidos nessas duas regiões. Ela tem apresentado predileção em sugar sangue fora de casa, embora um percentual moderado de exemplares possa ser encontrado no intradomicílio, assemelhando-se, neste aspecto, à população da Amazônia, em contraposição àquela do Nordeste brasileiro, onde, geralmente, apresenta grau elevado de endofilia 1.

As demais espécies ocorreram em baixa densidade ou irregularmente, comportando-se como espécies ocasionais. Dessas, A. argyritarsis, A. maculipes, A. minor e A. peryassui estiveram presentes nos municípios amazônicos (regiões noroeste e sudoeste) e litorâneos (nordeste), não sendo registradas nos municípios da região central.

Neste estudo, chamou a atenção a maior riqueza de espécies de anofelinos no noroeste e nordeste do estado, ambas as regiões influenciadas pelo litoral e compostas de uma rica hidrografia, o que certamente favorece a formação de numerosos e variados criadouros. A região noroeste tem influência amazônica, apresenta vegetação florestal e registra maior índice pluviométrico, tendendo a formar extensas áreas de campos inundáveis que persistem por toda a estação chuvosa e por grande parte da estação de estiagem, caracterizando o que se designa de Baixada Ocidental. No período deste tabalho, 
Distribuição das espécies de anofelinos na região sudoeste do Estado do Maranhão, Brasil, de 1993 a 2002.

\begin{tabular}{|c|c|c|c|c|c|c|c|c|c|}
\hline \multirow[t]{2}{*}{ Municípios } & \multicolumn{7}{|c|}{ Espécies do gênero Anopheles } & \multicolumn{2}{|c|}{ Total } \\
\hline & nuneztovari & oswaldoi & peryassui & rondoni & strodei & triannulatus & Anopheles sp. & Indivíduos & Espécies \\
\hline Açailândia & 71 & - & 3 & - & - & 160 & - & 465 & 6 \\
\hline Amarante & 819 & 3 & - & - & - & 1.254 & - & 3.732 & 6 \\
\hline Arame & 539 & 18 & - & - & - & 296 & - & 1.133 & 6 \\
\hline Barra do Corda & 174 & - & - & - & - & 683 & - & 1.296 & 4 \\
\hline Bom Jardim & 307 & 12 & - & - & - & 698 & - & 1.825 & 8 \\
\hline Bom Jesus das Selvas & 55 & 1 & - & - & - & 98 & - & 297 & 6 \\
\hline Buriticupu & 19 & - & - & 1 & - & 49 & - & 389 & 6 \\
\hline Buritirana & 50 & - & - & - & - & 43 & - & 220 & 5 \\
\hline Campestre & 93 & - & - & - & - & 125 & - & 528 & 4 \\
\hline Carolina & - & - & - & - & - & - & - & 93 & 1 \\
\hline Cidelândia & - & - & - & - & - & 4 & - & 9 & 4 \\
\hline Davinópolis & - & - & - & - & - & 6 & - & 48 & 4 \\
\hline Estreito & 3 & - & - & - & - & - & - & 64 & 4 \\
\hline Formosa Serra Negra & 12 & - & - & - & - & 58 & - & 118 & 5 \\
\hline Governador Edson Lobão & 239 & - & - & - & - & 284 & - & 703 & 4 \\
\hline Grajaú & 90 & 2 & - & - & - & 110 & - & 627 & 6 \\
\hline Imperatriz & 1.004 & 13 & - & - & 8 & 1.769 & 6 & 6.187 & 11 \\
\hline Itinga & 11 & - & - & - & - & 51 & - & 374 & 5 \\
\hline João Lisboa & 3 & 2 & - & - & - & 34 & - & 69 & 6 \\
\hline Montes Altos & 4 & - & - & - & - & 46 & - & 86 & 5 \\
\hline Porto Franco & - & - & - & - & - & 97 & - & 180 & 2 \\
\hline Ribamar Fiquene & 15 & 1 & - & - & - & 112 & - & 353 & 6 \\
\hline São Francisco do Brejão & - & - & - & - & - & 4 & - & 23 & 3 \\
\hline São João do Paraíso & - & - & - & - & - & 31 & - & 31 & 1 \\
\hline São Pedro da Água Branca & 333 & - & - & - & - & 126 & - & 915 & 5 \\
\hline São Pedro dos Crentes & 3 & - & 6 & - & - & 13 & - & 147 & 6 \\
\hline Senador La Roque & - & - & - & - & 64 & 240 & - & 5 & - \\
\hline Sítio Novo & 9 & 3 & - & - & - & 69 & - & 132 & 6 \\
\hline Total de indivíduos & 3.857 & 55 & 9 & 1 & 8 & 6.284 & 6 & 20.284 & 16 \\
\hline Total de municípios & 22 & 9 & 2 & 1 & 1 & 26 & 1 & 28 & - \\
\hline
\end{tabular}

essa região contribuiu com os maiores índices de positividade anual (IPA) de malária, variando entre 2,52 e 79,85, de acordo com os municípios. Já a região nordeste, apesar da rica hidrografia e de constituir a Baixada Oriental, é influenciada pelo sertão, o que pode ser notado não apenas pela redução da precipitação pluviométrica, como também pelas características da vegetação, predominando a associação de mata aberta, cerrado e manchas de caatinga. Ainda assim, quatro espécies (A. antunesi, A. apicimacula, A. pseudomaculipes e A. shannoni) foram encontradas exclusivamente nessa região. Essas características, de transição climática e vegetacional, talvez favoreçam a sobreposição de espécies de ambas as áreas, úmidas e xéricas, resultando numa fauna anofélica heterogênea. O IPA nessa região variou menos do que na região noroeste, na ordem de 0,11 a 26,97.

A diversidade de espécies também foi elevada na região sudoeste, a qual também tem influência amazônica. A exemplo das outras regiões, o predomínio de $A$. darlingi juntamente com $A$. albitarsis s.l., A. nuneztovari, A. triannulatus s.l. e $A$. evansae justifica o IPA encontrado, variando entre 0,04 e 28,24, a depender do município considerado.

Na região central, onde predomina a mata dos cocais, houve uma redução sensível na diversidade de espécies. Essa região tem clima de transição e, por situar-se no alto curso do rio Mearim, apresenta um menor volume de água quando comparada com as áreas de baixadas das regiões noroeste e nordeste do estado. Ape- 
Distribuição das espécies de anofelinos da região central do Estado do Maranhão, Brasil, 1993 a 2002.

\begin{tabular}{|c|c|c|c|c|c|c|c|c|c|c|}
\hline \multirow[t]{2}{*}{ Municípios } & \multicolumn{8}{|c|}{ Espécies do gênero Anopheles } & \multicolumn{2}{|c|}{ Total } \\
\hline & albitarsis & darlingi & evansae & galvaoi & intermedius & nuneztovari & oswaldoi & triannulatus & Indivíduos & Espécies \\
\hline Alto Alegre & 345 & 284 & - & - & 1 & 1.081 & 4 & 911 & 2.626 & 6 \\
\hline Arari & 213 & 70 & 143 & 1 & - & 77 & - & 260 & 764 & 6 \\
\hline Bacabal & 461 & 699 & 1 & - & - & 932 & - & 382 & 2.475 & 5 \\
\hline Bela vista & 63 & 107 & - & - & - & 11 & - & 34 & 215 & 4 \\
\hline Conceição do Lago Açu & 261 & 1.729 & - & - & - & 1.438 & - & 508 & 3.936 & 4 \\
\hline Coroatá & 324 & 416 & - & - & - & 995 & - & 536 & 2.271 & 4 \\
\hline Esperantinópolis & 24 & 8 & 3 & - & - & 198 & - & 125 & 358 & 5 \\
\hline Governador Eugênio Barros & - & 10 & - & - & - & 8 & - & 18 & 36 & 3 \\
\hline Igarapé do Meio & 147 & 47 & - & - & - & 91 & - & 306 & 591 & 4 \\
\hline Igarapé Grande & 51 & 303 & - & - & - & 42 & - & 67 & 463 & 4 \\
\hline Joselândia & 229 & 106 & - & - & - & 107 & - & 1 & 443 & 4 \\
\hline Lago do Junco & 5 & - & - & - & - & 17 & - & 34 & 56 & 3 \\
\hline Lago Verde & 121 & 150 & - & - & - & 130 & - & 111 & 512 & 4 \\
\hline Lima Campos & 27 & 12 & - & - & - & 9 & - & 59 & 107 & 4 \\
\hline Olho Dágua das Cunhas & - & 8 & - & - & - & - & - & - & 8 & 1 \\
\hline Pedreiras & 27 & 47 & - & - & - & 268 & - & 394 & 736 & 4 \\
\hline Peritoró & 284 & 304 & - & - & - & 605 & - & 411 & 1.604 & 4 \\
\hline Pio XII & 330 & 319 & - & - & - & 503 & - & 179 & 1.331 & 4 \\
\hline Poção de Pedra & 8 & - & - & - & - & 16 & - & 7 & 31 & 3 \\
\hline Santa Inês & 58 & 54 & 9 & - & - & 20 & - & 78 & 219 & 5 \\
\hline Santa Luzia & 67 & 821 & 188 & - & - & 516 & - & 877 & 2.469 & 5 \\
\hline Santo Antonio do Lopes & 141 & 3 & - & - & - & 35 & - & 108 & 287 & 4 \\
\hline São Luís Gonzaga & 348 & 358 & 23 & - & - & 976 & - & 933 & 2.638 & 5 \\
\hline São Mateus & 596 & 1.589 & - & - & - & 1.488 & - & 1.037 & 4.710 & 4 \\
\hline Timom & 41 & 47 & - & - & - & - & - & 88 & 176 & 3 \\
\hline Trizidela do Vale & 495 & 1 & - & - & - & 62 & - & 211 & 769 & 4 \\
\hline Tufilândia & 54 & 51 & - & - & - & 67 & - & 170 & 342 & 4 \\
\hline Vitorino Freire & 72 & 199 & - & - & - & 210 & - & - & 481 & 3 \\
\hline Números de indivíduos & 4.792 & 7.742 & 364 & 1 & 1 & 9.902 & 4 & 7.845 & 30.654 & 8 \\
\hline Números de municípios & 26 & 26 & 6 & 1 & 1 & 26 & 1 & 26 & 28 & - \\
\hline
\end{tabular}

sar disso, o IPA encontrado variou entre $0,03 \mathrm{e}$ 26,91, de acordo com os municípios.

De modo geral, observou-se a ocorrência de várias espécies representadas por pouquíssimos indivíduos, ao lado de espécies como A. darlingi, A. albitarsis s.l., A. nuneztovari, A. triannulatus s.l. e A. evansae, que contribuíram com milhares de exemplares, em igual período. Essas diferenças devem estar relacionadas à própria estrutura da comunidade anofélica local e ao fato de o estudo ter sido realizado em áreas antrópicas, isto é, os inquéritos entomológicos da Fundação Nacional de Saúde são programados para serem executados nas habitações ou em suas proximidades. Esse aspecto deve ser considerado, pois nem todas as espécies de anofelinos existentes numa determinada região devem ser capazes de se aproximar das habitações humanas, a não ser ocasionalmente, restringindo sua ocorrência aos ambientes silvestres. Conforme as observações de Deane et al. ${ }^{1}$, A. intermedius e $A$. rangeli revelam maior afinidade com animais de grande porte e só costumam ser encontradas dentro das habitações humanas quando estas estão na floresta ou em sua proximidade; do mesmo modo, A. mediopunctatus e A. shannoni demonstram nítida preferência por copas de árvores, apesar de suas larvas se criarem em poças ou córregos; já A. argyritarsis prefere se alimentar em animais, especialmente os silvestres, e, no caso de A. eiseni e A. peryassui, estas costumam 
se alimentar ao ar livre, em campo aberto. Estes são apenas alguns exemplos.

Quanto ao papel dos anofelinos como vetores de malária, desde a década de 80 , vários estudos realizados na Amazônia, baseados em dissecações, ensaios imunológicos e no comportamento antropofílico, têm apontado o A. darlingi como principal vetor nessa região 29 , revelando-se capaz de manter a endemicidade palúdica em grau elevado mesmo quando presente em baixa densidade 1 . Na zona litorânea, $A$. aquasalis adquire importância e, em relação às outras espécies, relegadas a um plano secundário, é preciso lembrar que aquelas dos complexos $A$. triannulatus s.l. e A. albitarsis s.l., além de A. nunneztovari e A. oswaldoi, já foram encontradas infectadas com plasmódios humanos 13,30,31,32. Por isso, é importante determinar com exatidão os tipos desses complexos presentes no Maranhão, pois não se pode subestimá-los, principalmente em virtude de sua presença freqüente, do desenvolvimento da endofilia e da capacidade de se alimentar no homem. Tais características poderiam torná-los vetores palúdicos locais.

Em síntese, os dados apresentados neste estudo mostram claramente a existência de uma grande diversidade de espécies no Maranhão, muitas delas amplamente distribuídas no estado. Obviamente, esse padrão de diversidade e distribuição pode ser explicado pela interação de um conjunto de fatores, entretanto chama-se a atenção para a posição geográfica do estado, entre as macrorregiões que caracterizam o Brasil, associada com a riqueza de ecossistemas, diversidade de bacias hidrográficas e de tipos climáticos favoráveis, especialmente os de influência amazônica. Esses aspectos ajudam a entender por que a fauna anofélica maranhense, por exemplo, é mais rica do que aquela descrita em diversos estados nordestinos 10 e no extremo Sul do Brasil 15 e por que é quantitativamente similar àquelas encontradas nos estados do $\mathrm{Pa}$ rá 13 e Mato Grosso 14, também de influência amazônica.

\section{Resumo}

Estudou-se a distribuição e diversidade de espécies de Anopheles em 123 municípios do Estado do Maranhão, Brasil. O método básico foi a captura de fêmeas dentro e nos arredores das habitações humanas, em intervalos compreendidos entre $18 \mathrm{~h}$ e $6 \mathrm{~h}$, no período de janeiro de 1992 a dezembro de 2001. Foram capturados 84.467 exemplares distribuídos em 24 espécies, com o predomínio de A. triannulatus sensu lato (20.788), A. darlingi (19.083), A. nuneztovari (16.884), A. albitarsis s.l. (14.352), A. aquasalis (8.202) e A. evansae (2.885). As outras 18 espécies juntas representaram apenas 2,7\%. As espécies encontradas no maior número de municípios foram: A. albitarsis s.l. (109 municípios), A. triannulatus s.l. (106), A. nuneztovari (93), A. darlingi (87) e A. evansae (64). A riqueza e a ampla distribuição das espécies de anofelinos no Maranhão concordam com a posição geográfica do estado, entre as macrorregiões que caracterizam o Brasil, resultando em uma fauna mista, com elementos representativos dessas regiões.

Anopheles; Malária; Demografia

\section{Colaboradores}

W. A. Mendes, E. Costa, L. E. M. B. Câmara, J. L. P. Moraes, G. A. Alves, F. S. Leonardo, R. V. Rocha, Y. N. O. Pereira, J. A. C. Mendonça e M. J. A. Silva participaram do planejamento das atividades, coleta e identificação dos anofelinos, da supervisão dos trabalhos e da revisão do manuscrito. Coube a J. M. M. Rebêlo a coleta de anofelinos, análise dos dados e elaboração do artigo. 


\section{Referências}

1. Deane LM, Causey OR, Deane MP. Notas sobre a distribuição e a biologia dos anofelinos das regiões nordestina e amazônica do Brasil. Rev Serv Esp Saúde Pública 1948; 1:967-76.

2. Ferreira Neto J, Deane LM, Carneiro EWB. Infecção natural de guaribas, Alouatta belzebul (L., 1766) pelo Plasmodium brasilianum (Gonder \& Berenberg-Grossler, 1908), no Estado do Maranhão, Brasil. Rev Inst Med Trop São Paulo 1970; 12:169-74.

3. Xavier SH, Mattos SS. Lista das espécies e gêneros de culicídeos encontrados nos estados do Brasil. IX. Maranhão (Diptera, Culicidae). Acta Amaz 1989; 19:295-306.

4. Rebêlo JMM, Silva AR, Ferreira LA, Vieira JA. Anopheles (Culicidae, Anophelinae) e a malária em Buriticupu, pré-Amazônia Maranhense. Rev Soc Bras Med Trop 1997; 30:107-11.

5. Xavier MMP, Rebêlo JMM. Espécies de Anopheles (Culicidae, Anophelinae) em área endêmica de malária, Maranhão, Brasil. Rev Saúde Pública 1999; 33:535-41.

6. Pereira YN, Rebêlo JMM. Espécies de Anopheles no Município de Pinheiro (Maranhão), área endêmica de malária. Rev Soc Bras Med Trop 2000; 33:44350.

7. Instituto Brasileiro de Geografia e Estatística. Atlas do Maranhão. Rio de Janeiro: Instituto Brasileiro de Geografia e Estatística/São Luís: Governo do Estado do Maranhão; 1984.

8. Forattini OP. Entomologia médica. v. 1. São Paulo: Faculdade de Higiene e Saúde Pública, Universidade de São Paulo; 1962.

9. Consoli RAGB, Lourenço-de-Oliveira R. Principais mosquitos de importância sanitária no Brasil. Rio de Janeiro: Editora Fiocruz; 1994.

10. Deane LM. Malaria vectors in Brazil. Mem Inst Oswaldo Cruz 1986; 81:5-14.

11. Tadei WP, Santos JMM, Costa WLS, Scarpassa VM. Biologia de anofelinos amazônicos. XII - Ocorrência de espécies de Anopheles, dinâmica de transmissão e controle da malária na zona urbana de Ariquemes (Rondônia). Rev Inst Med Trop São Paulo 1988; 30:221-51.

12. Xavier SH, Mattos SS, Calábria PV, Cerqueira E. Geographical distribution of Culicinae in Brazil - VII. State of Ceará (Diptera, Culicidae). Mosquito Systematics 1983; 15:127-40.

13. Tadei WP, Tatcher BD. Malaria vectors in the brazilian amazon: Anopheles of the subgenus Nyssorhynchus. Rev Inst Med Trop São Paulo 2000; 42:87-94.

14. Xavier SH, Mattos SS. Distribuição geográfica dos culicíneos no Brasil (Diptera, Culicidae). II. Estado de Mato Grosso. Rev Bras Malariol Doenças Trop 1970; 22:441-60.

15. Cardoso JC, Corseuil E, Barata JMS. Anophelinae (Díptera, Culicidae) ocorrentes no Estado do Rio Grande do Sul, Brasil. Entomol Vectores 2004; 11:159-77.

16. Rosa-Freitas MG, Lourenço-de-Oliveira R, Carvalho-Pinto CJ, Flores-Mendoza C, Silva-do-Nascimento TF. Anopheline species complexes in Brazil. Current knowledge of the related to malaria transmission. Mem Inst Oswaldo Cruz 1998; 93:651-5.
17. Silva-do-Nascimento TF, Lourenço-de-Oliveira, R. Anopheles halophylus, a new species of the subgenus Nyssorhynchus (Diptera: Culicidae) from Brazil. Mem Inst Oswaldo Cruz 2002; 97:801-11.

18. Klein TA, Lima JBP, Tada MS. Comparative susceptibility of anopheline mosquitoes to Plasmodium falciparum in Rondônia, Brazil. Am J Trop Med Hyg 1991; 44:598-603.

19. Klein TA, Lima JBP, Tada MS, Miller R. Comparative susceptibility of anopheline mosquitoes in Rondônia, Brazil, to infection by Plasmodium vivax. Am J Trop Med Hyg 1991; 45:463-70.

20. Li C, Wilkerson RC. Identification of Anopheles (Nyssorhynchus) albitarsis complex species (Diptera: Culicidae) using rDNA internal transcribed spacer 2-based polymerase chain reaction primes. Mem Inst Oswaldo Cruz 2005; 100:495-500.

21. Wilkerson RC, Pearsons TJ, Klein TA, Gaffigan TV, Bergo E, Consolim J. Diagnosis by random amplified polymorphic DNA polymerase chain reaction of four cryptic species related to Anopheles albitarsis from Paraguay, Argentina and Brazil. J Med Entomol 1995; 32:697-704.

22. Conn JE, Rangel-Puertas Y, Seawright J. A new cytotype of Anopheles nuneztovari from western Venezuela and Colombia. J Am Mosq Control Assoc 1993; 9:294-301.

23. Fritz GN, Conn J, Cockburn A, Seawright J. Sequence analyses of the ribosomal DNA internal transcribed spacer 2 from populations of Anopheles nuneztovari (Diptera: Culicidae). Mol Biol Evol 1994; 11:406-16.

24. Linley JR, Lounibos LP, Conn J, Duzak D, Nishimura N. A description and morphometric comparison of eggs from eight geographic populations of the South American malaria vector Anopheles (Nyssorhynchus) nuneztovari. J Am Mosq Control Assoc 1996; 12:275-92.

25. Sallum MAM, Bergo ES, Flores DC, Forattini OP. Systematic studies on Anopheles galvaoi Causey, Deane \& Deane from the subgenus Nyssorhynchus blanchard (Diptera: Culicidae) Mem Inst Oswaldo Cruz 2002; 97:1177-89.

26. Faran ME. Mosquito studies (Diptera: Culicidae). XXXIV - A revision of the albimanus section of the subgenus Nyssorhynchus of Anopheles. Contributions of the American Entomological Institute $1980 ; 15: 1-215$.

27. Lopes J, Lozovei AL. Ecologia de mosquitos (Diptera, Culicidae) em criadouros naturais e artificiais de área rural do norte do Estado do Paraná, Brasil. I - Coletas ao longo do leito de ribeirão. Rev Saúde Pública 1995; 29:183-91.

28. Tadei WP, Tatcher BD, Santos JMM, Scarpassa VM, Rodrigues IB, Rafael MS. Ecologic observations on Anophelinae vectors of malaria in the Brazilian Amazon. Am J Trop Med Hyg 1998; 59:325-35.

29. Póvoa MM, Wirtz RA, Lacerda RNL, Miles MA, Warhurts D. Malaria vectors in the Municipality of Serra do Navio, State of Amapá, Amazon Region, Brazil. Mem Inst Oswaldo Cruz 2001; 96:179-84. 
30. Arruda ME, Carvalho MB, Nussenzweig RS, Maracic M, Ferreira AW, Cochrane AH. Potential vectors of malaria and their different susceptibility to Plasmodium falciparum and Plasmodium vivax in northern Brazil identified by immunoassay. Am J Trop Med Hyg 1986; 35:873-81.
31. Branquinho MS, Araújo MS, Natal D, Marelli MT, Rocha RM, Taveira FAL, et al. Anopheles oswaldoi an important potential malaria vector in Acre, Brazil. Trans R Soc Trop Med Hyg 1996; 90:233.

32. Oliveira-Ferreira J, Lourenço-de-Oliveira R, Teva A, Deane LM, Daniel-Ribeiro CT. Natural malaria infections in anophelines in Rondônia State, Brazilian Amazon. Am J Trop Med Hyg 1990; 43:6-10.

Recebido em 14/Mar/2006

Versão final reapresentada em 24/Abr/2007

Aprovado em 07/Mai/2007 